

Originally published as:

Ditommaso R., Mucciarelli M., Ponzo F. C. (2012). ANALYSIS OF NONSTATIONARY STRUCTURAL SYSTEMS BY USING A BAND-VARIABLE FILTER. Bulletin of Earthquake Engineering. DOI: 10.1007/s10518-012-9338-y. 


\title{
ANALYSIS OF NON-STATIONARY STRUCTURAL SYSTEMS BY USING A BAND- VARIABLE FILTER
}

\author{
Rocco DITOMMASO, Marco MUCCIARELLI, Felice Carlo PONZO \\ DiSGG, University of Basilicata, Potenza, Italy
}

Keywords: Non-Parametric Dynamic Identification - Structural Health Monitoring Nonlinear Systems - Non-Stationary Systems - S-Transform - Strong Motion

\begin{abstract}
One of the main tools used to study the dynamic response of structural systems is certainly the Fourier Transform. This tool is very useful and reliable to investigating the response of a stationary system, i.e. a generic system that does not changes its characteristics over time. Conversely, the Fourier Transform is no longer reliable if the main goal is to study the evolution of the dynamic response of a system whose features rapidly vary with time. To this regard, several mathematical tools were developed to analyze time-variable dynamic responses. Soil and buildings, subject to transient forcing such as an earthquake, may change their characteristics over time with the initiation of nonlinear phenomena. This paper proposes a new methodology to approach the study of non-stationary response of soil and buildings: a band-variable filter based on S-Transform. In fact, with the possibility of changing the bandwidth of each filtering window over time, it becomes possible to extract from a generic record only the response of the system focusing on the variation of individual modes of vibration. Practically, it is possible to extract from a generic non-stationary signal only the phase of interest. The paper starts from examples and applications on synthetic signals, then examines possible applications to the study of the non-stationary response of soil and buildings. The last application focuses on the possibility to evaluate the mode shapes over time for both numerical and scaled model subjected to strong motion inputs.
\end{abstract}

\section{INTRODUCTION}

In the past years, several techniques for signal analysis and structural dynamic identification were proposed with the aim to evaluate the dynamic characteristics of soil and structures. One of the main tools used in the past years is the Fourier Transform (e.g., Chopra, 1995) and some tools derived from the same such as Transfer Function and others techniques based on transformations from time to frequency domain (Fotsch et al., 2000; Clough and Penzien, 
2003; Snieder and Şafak, 2006). In recent years many authors have proposed techniques to overcome some of the limitations of the classical analyses when signals are non-stationary (Gabor, 1946; Cohen, 1989; Young, 1993; Addison, 2002; Guan et al., 2005; Dehghani, 2009; Rainieri and Fabroccino, 2010). For structural engineers, non-stationarity in the seismic signal recorded within a building is generally linked to the variability of the input (i.e. earthquake, explosion, etc..), to the non-linear behaviour of the structure (i.e. damage evolution), to the dynamic interaction between structure and soil or adjacent structures.

The study of the evolution over time of dynamic characteristics of a system has often been addressed in an approximate way, using tools that require the assumption of stationarity, and these techniques might not be appropriate. For example, one of the main tools used to analyze dynamical systems is the Fourier transform, which for a function $h(t)$ holds:

$$
H(f)=\int_{-\infty}^{+\infty} h(t) \cdot e^{-\mathrm{i} \cdot 2 \cdot \pi \cdot f \cdot t} d t
$$

This technique, as well as all those tools which found their basis on the assumption of stationary behaviour of the system, appears to be not appropriate when studies on system that changes its characteristics over time. In fact, evaluating the Fourier Transform of a nonstationary signal, we obtain just an average of each spectral amplitude assessed on the entire length of the analyzed signal, loosing information about the local spectrum and then on the time varying behaviour.

In order to overcome some of the limitations derived from the Fourier Transform, another tool widely used to study the transients is the STFT (Short Time Fourier Transform) (Gabor, 1946). This technique exceeds certain limits of the simple Fourier transform, giving some indications on the variation over time of the spectral characteristics of analyzed signal. For a signal $h(t)$ the SFTF is defined as:

$$
\operatorname{STFT}(\tau, f)=\int_{-\infty}^{+\infty} h(t) \cdot w(t-\tau) \cdot e^{-\mathrm{i} \cdot 2 \cdot \pi \cdot f \cdot t} d t
$$

where $w(t-\tau)$ is a generic moving window. Unfortunately, also this integral transformation has some limits which tend to distort the result. Indeed, the values of the moving-averaged spectral magnitudes have no absolute value, but may be used only for relative comparing over time. In order to assess the STFT it is necessary to choose the length of the time-window. This choice cannot be done arbitrarily, but is bound by the necessity of having a good resolution both in time and in frequencies domain. To have a good time resolution, a very short time-window should be chosen, but in this case, a very low resolution in the frequency 
domain could be obtained. On the contrary, a very long time-window could be selected in order to have a good frequency resolution. In the end, depending on the problem that one wants to address, it is necessary to do a suitable choice of the time-window length.

More recently, several techniques for time-frequency signals analysis have been developed. The most widely used are the Wavelet Transform (Daubechies, 1992) and the Wigner-Ville Distribution (Wigner, 1932; Ville, 1948; Bradford et al., 2006). These transformations, for a signal $h(t)$ are defined respectively as:

$$
F(a, b)=|a|^{-\frac{1}{2}} \int_{-\infty}^{+\infty} h(t) \cdot w^{*}\left(\frac{t-b}{a}\right) d t
$$

where $a$ and $b$ are two scale factors and $w^{*}$ is a generic moving window,

$$
W D(\tau, f)=\int_{-\infty}^{+\infty} h\left(\tau+\frac{t}{2}\right) \cdot h\left(\tau-\frac{t}{2}\right) \cdot e^{-i \cdot 2 \cdot \pi \cdot f \cdot t} d t
$$

where $t$ is time, $f$ is frequency and $\tau$ is a parameter that controls the position of the function. These other transformations provide a number of advantages compared with the Fourier transform and the STFT, but do not allow a fair assessment of the local spectrum. In other words, these instruments are insufficient to a correct evaluation of the spectral characteristics taking into account the instantaneous variations of the local spectrum.

A tool that overcomes these limitations and allows to accurately assess both the spectral characteristics and their local variation over time is the S transform (Stockwell et al., 1996). This transformation, for a signal $h(t)$, is defined as:

$S(\tau, f)=\frac{|f|}{2 \pi} \int_{-\infty}^{+\infty} h(t) \cdot e^{-\frac{(\tau-t)^{2} \cdot f^{2}}{2}} \cdot e^{-i \cdot 2 \cdot \pi \cdot f \cdot t} d t$

where $t$ is time, $f$ is frequency and $\tau$ is a parameter controlling the position of Gaussian window along the time axis. As it has been defined in the transformation, the properties of Gaussian window scale derive from the continuous wavelet (Mallat, 1998). However, in this case the constrain of zero mean for the wavelets it is not requested. Furthermore, compared to the wavelet transform, the S-Transform changes the shape of the real and imaginary coefficients over time together with the temporal translation of the Gaussian window. On the contrary, the wavelet transform does not have this property: the entire waveform translates over time, but never changes its shape (Parolai, 2009). The S-Transform also has many important properties, for example, the S-Transform can be written as an operator that is a function of the Fourier spectrum (Stockwell et al., 1996): 
$S(\tau, f)=\frac{|f|}{2 \pi} \int_{-\infty}^{+\infty} H(\alpha+f) \cdot e^{-\frac{2 \cdot \pi^{2} \cdot \alpha^{2}}{f^{2}}} \cdot e^{-i \cdot 2 \cdot \pi \cdot \alpha \cdot \tau} d \alpha \quad$ with $f \neq 0$

Furthermore, it can be shown that (Stockwell et al., 1996):

$H(f)=\int_{-\infty}^{+\infty} S(\tau, f) d \tau$

So it is possible to completely recover the function $h(t)$ by using the following relation (Stockwell et al., 1996):

$h(t)=\int_{-\infty}^{+\infty}\left(\int_{-\infty}^{+\infty} S(\tau, f) d \tau\right) \cdot e^{i \cdot 2 \cdot \pi \cdot f \cdot t} d f$

The last, but not least important property is the linearity of the S-Transform (Stockwell et al., 1996):

$S\{$ data $(t)\}=S\{$ signal $(t)\}+S\{$ noise $(t)\} \quad$ where $S$ stands for $S$-Transform

Thanks to this property it is possible to extract the processed information from the signal of interest, without modifying the local spectra characteristics. Taking advantage of these properties, the S-Transform has been used up to now in seismology and earthquake engineering to clean signals from unwanted noise. Examples of the use of S-Transform to enhance the signal to noise ratio are given in Pinnegar and Eaton (2003); Schimmel and Gallart (2005); Ascari and Siahkoohi (2007); Simon et al. (2007); Parolai (2009); Ditommaso et al. (2010c). The main goal of this paper is to provide a band-variable filter able to extract from a non-stationary signal only the phase of interest. It will be discussed the possibility on the use of the band-variable filter to extract from a signal recorded on a structure (excited by an earthquake) the response related to a single mode of vibration for which the related frequency changes over time if the structure is being damaged.

\section{BAND-VARIABLE FILTER (BV-Filter)}

This paper discusses the possibility to use the S-filter to extract the dynamic characteristics of systems that evolve over time by acting simultaneously in both frequency and time domain. The filter was built using the properties of convolution, linearity and invertibility of the STransform. The filtering method, here discussed from the mathematical point of view, is based on the algorithm described in the following steps:

1- Assessment of S-Transform $S(\tau, f)$ of the signal $h(t)$;

2- Generating the filtering matrix $G(\tau, f)$, selecting the time-frequency subdomain directly from the S-Transform result; 
3- Calculating the convolution in the time-frequency domain $M(\tau, f)=G(\tau, f) * S(\tau, f)$;

4- Retrieving the filtered signal $h_{f}(t)$ through the calculation of the inverse S-transform matrix $M(\tau, f)$.

So the complete process can be written as:

$h_{f}(t)=\int_{-\infty}^{+\infty}\left(\int_{-\infty}^{+\infty}[S(\tau, f) * G(\tau, f)] d \tau\right) \cdot e^{i \cdot 2 \cdot \pi \cdot f \cdot t} d f$

A generic $k$-th column $G\left(\tau_{k}, f\right)$ identifies a filtering window at a given time step. In order to better clarify the filtering procedure a worked example will be provided in the next section. Defining the filtering matrix could be a time-demanding operation, since each filtering window should be defined over a varying frequency recursively with a fixed time lapse. Moreover, the band-pass portion of the filter must be enlarged or reduced for each time step according to the desired bandwidth and the rectangular shape of the matrix. In order to simplify this process, it is convenient to try to automate it. We propose an implementation that defines the filtering matrix using a graphical user interface (GUI) that visualizes the amplitude spectra of the S-transform of the signal allowing for a point-and-click selection of the desired portion of the time-frequency domain. The routine is designed so that the user can select a few points and the computer performs a cubic spline interpolation. In order to build the filtering matrix we used the pimf function of MATLAB $\odot$, which is a convenient approximation of a boxcar function. This spline-based curve is so named because of its $\Pi$ shape. It is possible to obtain more details about this function from www.mathworks.com. Figure 1 shows an example of pimf function used to build the filtering matrix $G(\tau, f)$.

\section{APPLICATION ON SYNTHETIC DATA}

The first application regards a synthetic signal composed by the superposition of a signal with constant amplitude and slowly changing frequency over time, and a signal with stationary frequency but with varying amplitude. The signal length is equal to $22 \mathrm{sec}$ with $1 \mathrm{sec}$ of zeros padding at beginning and end to avoid numerical stability problems. The varying-frequency, constant amplitude signal start at $11 \mathrm{~Hz}$, in the central part the frequency reaches the minimum value, equal to $3 \mathrm{~Hz}$, then it returns to a frequency equal to $11 \mathrm{~Hz}$. The frequency variation follows a parabolic function. The signal is plotted in Figure 2a. The constantfrequency, varying amplitude signal has a frequency equal to $9 \mathrm{~Hz}$ and amplitude equal to zero at the begin and at the end, and equal to the other signal in the middle of the time span considered (Figure 2b). The result is a strong disturbance in the central part, corresponding to 
the minimum values of the frequency of the main signal. It should be noted that the frequency of the disturbance is contained within the range of variation of the main signal, and this could be an insurmountable problem when using a classical approach.

Figure $3 a$ reports the sum of the signals, while Figure $3 b$ shows the Fourier spectrum of the signal. It is evident from the spectrum shown in Figure 3b, that looking only at the Fourier spectrum it is impossible to distinguish the two signals, and using a traditional filter, it would be impossible to eliminate the interference without affecting the original signal. In this case a variable band-pass filter is helpful to isolate the first signal from the second one. From Figure 4a the advantage of using the S-Transform in place of the classical Fourier transform is evident: it is possible to clearly distinguish the two signals and the variation of the energy content over time. From the S-Transform of the signal it is possible to select the subdomain of interest (red curve depicted in Figure 4a) and then create the filtering matrix using spectral amplitude equal 1 within the selected domain and zero out of the selected subdomain as showed in Figure 4b. Using the selected subdomain, as discussed in the previous section, a generic $k$-th column $G\left(\tau_{k}, f\right)$ identifies a filtering window for a single time step. In this work, as discussed above, each filtering window $G\left(\tau_{k}, f\right)$ has been modelled using a pimf function (Figure 1). Figure $4 \mathrm{c}$ shows the signal retrieved from the variable band filter together with its S-transform. Similarly to what was done to extract the variable frequency signal, it is possible to extract the component that currently is called disturbance, i.e. the frequency-constant, timevarying amplitude.

It is important to underline that the values used to construct the filtering matrix are real numbers, and then the part of the complex matrix resulting from the calculation of the STransform is not altered. The result is a filtering matrix that changes the amplitude without altering the phases of the signal. So, a variable band-pass filter that gives a filtered signal with phases corresponding to those of the original signal.

\section{APPLICATIONS ON REAL DATA}

In the following we show three real case histories that illustrate how it is possible to extract from a real recording the information of interest: the first signal was recorded on the ground during an experimental campaign on a full-scale structure; the second one was recorded on a building while it was being damaged by an aftershock of the 2002, Molise earthquake (Italy); the third one was recorded during the 1995, Kobe earthquake at a site where soil suffered severe non-linearity leading to liquefaction. We retrieve the original data, and for all of them we applied standard corrections, removing both the baseline and possible trends. No band- 
pass filters were applied to preserve the original information as much as possible. Furthermore, specific details about data sources and processing are given in the following when necessary.

The first case, the Bagnoli experiment, is a controlled demolition experiment (Dolce et al. 2006) developed in order to test several shape-memory devices for retrofitting buildings. During the release tests on the two stories RC structure it was also possible to study the seismic waves released from the oscillating building. Several release tests were carried out on the monitored building that was initially moved from its initial position, thanks to a hydraulic jack contrasted against a steel reaction frame, and then left moving in free oscillations. In order to monitor the behaviour of the structure equipped with various devices, several control devices have been installed on the building and on the surrounding area. Thanks to the presence of the accelerometers installed in the surrounding area it was possible to record the ground motion caused by the oscillating building. On the ground the signals were recorded using a sampling frequency equal to $100 \mathrm{~Hz}$. Previous work (Gallipoli et al. 2006; Ditommaso et al. 2010a) showed how the seismic waves generated by the experimental system had two dominant frequencies: one at about $20 \mathrm{~Hz}$ due to the contrast steel frame and one at about 1-2 $\mathrm{Hz}$ due to the main structure. This second frequency was variable due to the non-linear and dissipative effect of the retrofitting braces. Figure 5 shows the displacements recorded in correspondence of the accelerometric station placed $10 \mathrm{~m}$ away from the building and both the S-Transform of the registration and the zoom in the frequency range 0-3 Hz.

The fundamental mode of vibration has been isolated from both higher modes and noise content using the band-variable filter. The result (Figure 6) shows that it is possible to extract from a real signal the local spectrum with higher energy content that is varying over time due to the change of stiffness of the system caused by the special behaviour of the shape-memory devices. Moreover, it is possible to avoid the phase distortions that can be introduced by standard causal filters: the superposition of original and band-variable filtered signal shows that both amplitude and phase are correctly preserved by the forward and inverse transformation provided by the S-Transform.

The second example is taken form the 2002 seismic sequence in Molise (Italy). The town of Bonefro endured little damage $\left(\mathrm{I}_{\mathrm{MCS}}=\mathrm{VII}\right)$ except for two reinforced concrete buildings that suffered severe damage (Mucciarelli et al., 2004). The buildings are very similar, both as design and construction, and are located near to each other in an area where the surface layers appear to be formed by soft sediments. The most damaged building has 4 stories, while the less damaged has 3 stories. As a result of the earthquake of the first of November (M 5.3) the 
taller building had an additional damage to structural parts. A seismometer located within the building recorded the seismic response of the building during the progression of damage (Mucciarelli et al. 2004) using a sampling frequency equal to $128 \mathrm{~Hz}$. The building exhibited a strong non-linear behaviour, leading to a $40 \%$ decrease of the frequency of the fundamental mode. Event recordings for the two orthogonal components in the horizontal plane are shown in Figure 7 with the respective S-Transform. Notwithstanding the saturation of the seismometer during the event, it is possible to follow the evolution of the dynamics of the building in the time-frequency domain. The attention was focused on the NS component of the recorded motion of building. Using the S-Transform analysis is evident that the building starts from a frequency equal to $1.9 \mathrm{~Hz}$, reaches the minimum frequency (around $1.2 \mathrm{~Hz}$ ) during the maximum excitation before recovering in the final part of the signal at about 1.8 Hz. At this point the response of the building was isolated by using the band-variable filter. The result of filtering is shown in Figure 8. It is interesting to note that, thanks to the selection of the local spectrum, the S-filter tool is able to reconstruct the dynamic response of the structure, with a negligible amount of noise even during non-stationary response. So, having at the disposal the recordings at all levels of the building, the behaviour of individual modes of vibration and their variation over time can be studied as it will be shown in the following chapter.

Finally, the band-variable filter was applied to extract the soil fundamental frequency during the 1995 Kobe earthquake (Japan). The site of Port Island experienced soil liquefaction and thus a large variation of stiffness for the surface layers, resulting in a variation of the fundamental frequency. The signals recorded at different depths during this earthquake have been extensively studied by Aguirre and Irikura (1997). The signal used in this example has been recorded using a sampling frequency equal to $100 \mathrm{~Hz}$.

Figure 9 reports the NS component of ground motion at the surface. Analyzing the STransform of the signal it is clear that most of the energy is concentrated in a single mode of vibration that has a strong frequency change after just one cycle. Following the frequency change, which is due to liquefaction of the subsoil, no stiffness recovery is observed, at least in this short period of time. Information about the characteristics of the first mode of vibration of the soil were recovered trying to remove from the registration all the noise and the response of potentials higher modes. S-Transform of the filtered signal and the comparison between the two signals is shown in Figure 10. It is evident that also in this case the filter is able to follow and extract the time-variable fundamental mode. 


\section{APPLICATION FOR MODE SHAPES EVALUATION}

In this section, we will discuss about the opportunity to use the band variable filter to evaluate the mode shapes during the non-stationary phase. In fact, as shown in the previous paragraphs using the S-Transform it is possible to estimate the dynamic characteristics of a generic system with a good resolution both in time and frequency domain. Here we show how, using the proposed band variable filter, it is possible to extract the mode shapes of a system also during the phase of maximum nonlinearity.

To this purpose, two different cases were considered: a nonlinear numerical model and a 1:4 experimental scaled model tested in the structural laboratory of University of Basilicata. The signals related to the numerical model have acquired using a sampling frequency equal to $100 \mathrm{~Hz}$ while those related to the experimental scaled model have been acquired using a sampling frequency equal to $400 \mathrm{~Hz}$.

The former is a nonlinear numerical model of an R/C regular building, five floors, four frames along the longitudinal direction $(\mathrm{X})$ and three frames in the transverse direction $(\mathrm{Y})$, having a rectangular plan, $15 \times 12 \mathrm{~m}$. The considered structure has been designed following the criteria of the Italian seismic code (OPCM 3431/2005) for high ductility class (CDA), high seismic intensity (PGA 0.35g) and for soft soil type D. The height of each storey is $3 \mathrm{~m}$, for a total height of the building equal to $15 \mathrm{~m}$. Software based on nonlinear finite element SAP2000 (Computers \& Structures) has been used to model the 3-D structure. Beams and columns have been modelled with frame elements, assuming 20MPa cylindrical strength of concrete and $430 \mathrm{MPa}$ yield strength of steel. In order to simulate a structural nonlinear behavior during a strong ground motion, link elements and plastic hinges have been used at the end of beam and column elements respectively. Link elements have a Takeda hysteretic behavior, while plastic hinges have an axial load-dependent one. It is possible to find more details about this numerical model in Ponzo et al. (2010).

The mode shapes related to the fundamental mode of vibration were extracted using the signals filtered by mean the band variable filter following these steps:

- evaluation of structural response at the last floor, where the fundamental mode is more clear;

- creation of the filtering matrix based on the S-Transform from the accelerometric recording;

- convolution of the filtering matrix with signal recorded at each level;

- evaluation of the mode shape over time. 
Figure 11 shows the S-Transform related to the accelerometric recording performed at the last level of the structural numerical model. The behavior of the structure is clearly nonstationary, with a change over time of the frequency of the fundamental mode. The starting frequency is $1.5 \mathrm{~Hz}$, the minimum frequency is $0.45 \mathrm{~Hz}$ and the final frequency is $0.95 \mathrm{~Hz}$.

Figure 12 shows the frequency evolution of the fundamental mode extracted by means of the band variable filter and the time-point from which the mode shapes were evaluated: Mode Shape A, B and C (starting frequency, minimum frequency and final frequency).

It is worth noting that using the standard approach it would have been possible to evaluate only the starting and final mode shapes, while using the band variable filter it is possible to evaluate also the mode shape related to the minimum frequency recorded during the maximum excursion in the plastic field.

Figure 13 shows the mode shapes evaluated over time as indicates in Figure 12: Mode Shape B has a curvature bigger than Mode Shapes A and C. It is known that mode curvature is strongly linked to the building damage during a seismic event (Pandey et al., 1991). Therefore, being able to evaluate the mode curvature during the maximum excursion in nonlinear field and isolating it from superimposed signals, we can achieve a better understanding of the mechanisms of damage as well as a more precise location of the damage on the structure.

The 4-storey, 1/4 scaled model, was designed for gravity loads only and was tested at the Structural Laboratory of the University of Basilicata in Potenza, within the POP project (Dolce et al., 2005), through mono-axial shaking table test (Figure 14).

The model was tested using two different conditions: fixed base (phase I) and with isolation system (phase II). With regards to the tests in the fixed-base configuration mentioned above, the effective peak acceleration of the table was progressively increased from $0.05 \mathrm{~g}$ up to $0.35 \mathrm{~g}$, using different spectral shapes. All floor displacements were measured through Temposonic digital transducers, fixed to an external steel reference frame. The floor accelerations were acquired through a system of horizontal servo accelerometers.

Following the same approach described for the numerical model also the fundamental mode shape related to the POP model has been evaluated. Figure 15 shows the S-Transform related to the accelerometric recording at the last level of the structure. The starting frequency is $1.4 \mathrm{~Hz}$, the minimum frequency is $0.90 \mathrm{~Hz}$ and the final frequency is $1.35 \mathrm{~Hz}$. Figure 16 shows the frequency evolution of the fundamental mode extracted by means of the band variable filter and the time-point from which the mode shapes were evaluated: Mode Shape A, B and $\mathrm{C}$ (starting frequency, minimum frequency and final frequency). 
In order to highlight the frequency evolution of the POP model a normalized S-Transform has been used. Figure 17 shows the mode shapes evaluated over time as indicates in Figure 16. It is worth noting that also for the experimental model the Mode Shape B, evaluated during the maximum excursion in the nonlinear field, has curvature bigger than Mode Shapes A and C. Also in this case the curvatures obtained from the mode shapes opportunely cleaned from the noise and the effects of higher modes thorough the S-Transform give the best information on the damage intensity and location. Moreover, the evaluation carried out on curvatures confirmed the location of the damage observed directly on the experimental model.

\section{CONCLUSIONS}

Dealing with signal analysis and dynamic characterization of systems means a constant search of mathematical tools that enable to study in detail the phase of non-stationary response of dynamic systems. This paper shows how classical techniques based on Fourier transform provide good results when the response of the system is stationary, but fail when the system exhibits a non-stationary, time-varying behavior. To hamper classical techniques, it is not necessary that a building reaches damage: even the non-stationarity of the input and the possible interaction with the ground and/or adjacent structures can show the inadequacy of classic techniques (Ditommaso et al. 2010b). In 1996, Stockwell introduced a new powerful tool for the signals analysis: the S-Transform. Compared with the classical techniques for the time-frequency analysis, this transformation shows a much better resolution and also offers a range of fundamental properties such as linearity and invertibility. By exploiting these properties, it was possible to develop a filter whose band varies both in time and in frequency domains, being very useful to study the characteristics of nonstationary signals. As discussed in the previous sections, this tool becomes necessary when one wants to isolate the response of individual, time-varying modes of vibration of soil and buildings (e.g., when their dynamic characteristics evolve over time as a result of seismic events). The ability to investigate the non-stationary response of soils and buildings opens new scenarios, giving the opportunity to explore new possibilities. For example, the ability to isolate individual modes of vibration of a building make possible to explore their variation over time, evaluating the change in mode curvature. It is known that this parameter is strongly linked to the building damage during a seismic event (Pandey et al. 1991). Therefore, being able to evaluate the mode curvature during the maximum excursion in nonlinear field and isolating it from superimposed signals, allows for a better understanding 
of the mechanisms of damage as well as for a more precise location of the damage on the structure.

\section{ACKNOLEDGEMENTS}

This study was partially developed within the DPC-RELUIS 2010-13 Project, funded by the Italian Department of Civil Protection. The authors are grateful to two anonymous reviewers for the critical and constructive comments.

\section{REFERENCES}

Addison, P. S. (2002). The illustrated wavelet transform handbook: introductory theory and applications in science, engineering, medicine and finance. IOP Publishing. ISBN 0750306920.

Aguirre, J., and K. Irikura (1997). Nonlinearity, Liquefaction, and Velocity Variation of Soft Soil Layers in Port Island, Kobe, during the Hyogo-ken Nanbu Earthquake. Bulletin of the Seismological Society of America, Vol. 87, No. 5, pp. 1244-1258, October 1997.

Askari, R., and H. R. Siahkoohi (2007). Ground roll attenuation using the S and x-f-k transforms. Geophys. Prospect., Vol. 55, pp. 1-10.

Bradford S Case, Yang Jing, Heaton Thomas (2006). Variations in the dynamic properties of structures: the Wigner-Ville distribution. Proceedings of the 8th U.S. National Conference on Earthquake Engineering. April 18-22, 2006, San Francisco, California, USA.

Chopra, A. K. (1995). Dynamic of Structures - Theory and Applications to Earthquake Engineering. Pearson Prentice Hall. ISBN 013156174X.

Cohen, L. (1989). Time-Frequency Distributions-A Review. PROCEEDINGS OF THE IEEE, VOL. 77, NO. 7, JULY 1989.

Clough, R. and Penzien, J. (2003). Dynamics of Structures. 3rd ed., Computers \& Structures, Inc.

Computers \& Structures, Inc. "SAP2000 - Integrated Software for Structural Analysis \& Design, Technical Reference Manual". (www. http://www.csiberkeley.com/).

Daubechies I. (1992). Ten Lectures on Wavelet. Society for Industrial and Applied Mathematics. ISBN 0898712742.

Dehghani, M. J. (2009). Comparison of S-transform and Wavelet Transform in Power Quality Analysis. World Academy of Science, Engineering and Technology, 50, 2009.

Ditommaso, R., M. Mucciarelli, M. R. Gallipoli and F. C. Ponzo (2010a). Effect of a single vibrating building on free-field ground motion: numerical and experimental evidences. Bulletin of Earthquake Engineering, Volume 8, Number 3, pp 693-703. DOI: 10.1007/s10518-009-9134-5.

Ditommaso, R., S. Parolai, M. Mucciarelli, S. Eggert, M. Sobiesiak and J. Zschau (2010b). Monitoring the response and the back-radiated energy of a building subjected to ambient vibration and 
impulsive action: the Falkenhof Tower (Potsdam, Germany). Bulletin of Earthquake Engineering, Volume 8, Number 3, pp 705-722. DOI: 10.1007/s10518-009-9151-4.

Ditommaso R, Marco Mucciarelli, Felice C. Ponzo (2010c). S-Transform based filter applied to the analysis of non-linear dynamic behaviour of soil and buildings. 14th European Conference on Earthquake Engineering. Proceedings Volume. Ohrid, Republic of Macedonia. August 30 September 03, 2010.

Dolce M, Cardone D, Di Cesare A, Moroni C, Nicoletti M, Ponzo FC, Nigro D (2005). Dynamic tests on a 1:4 scaled R/C existing building: comparison of several isolation systems. 9th Assisi, Kobe

Dolce M, Cardone D, Gallipoli M.R, Mucciarelli M, Nigro D, Ponzo F.C., Santarsiero G. (2006). Shape Memory Alloys Braces, in "Seismic upgrading of r.c. buildings by advanced techniquesthe ILVA-IDEM research project” (pp. 325-359). ISBN: 88-7699-038-0. MONZA: Polimetrica International Scientific Publisher (ITALY). Federico Mazzolani Coordinator \& ed.

Fotsch, D. and Ewins, D.J. (2000). Applications of MAC in the Frequency Domain. International Modal Analysis Conference, IMAC, pp. 1225-1231.

Gabor, D. (1946). Theory of communications. J. Inst. Electr. Eng, Vol. 93, pp. 429-457.

Gallipoli, M. R., M. Mucciarelli, F. Ponzo, M. Dolce, E. D’Alema, and M. Maistrello (2006). Buildings as a Seismic Source: Analysis of a Release Test at Bagnoli, Italy. Bulletin of the Seismological Society of America, Vol. 96, No. 6, pp. 2457-2464.

Guan, H.; Karbhari, V.M.; and Sikorski, C.S (2005). Time-Domain Output Only Modal Parameter Extraction and its Application. Proceedings of the 1st International Operational Modal Analysis Conference, Copenhagen, Denmark, pp. 577-584.

Young, R.K. (1993). Wavelet Theory and its Applications. Kluwer Academic Publishers, Dordrecht, 1993.

Mallat, S. (1998). A Wavelet Tour of Signal Processing. Academic, New York.

Mucciarelli, M., A. Masi, M. R. Gallipoli, P. Harabaglia, M. Vona, F. Ponzo, and M. Dolce (2004). Analysis of RC Building Dynamic Response and Soil-Building Resonance Based on Data Recorded during a Damaging Earthquake (Molise, Italy, 2002). Bulletin of the Seismological Society of America, Vol. 94, No. 5, pp. 1943-1953.

Pandey, A. K., M. Biswas, M. M. Samman (1991). Damage detection from changes in curvature mode shapes. Journal of Sound and Vibration, Vol. 145, Issue 2, pp. 321-332.

Parolai, S. (2009). Denoising of Seismograms Using the S Transform. Bulletin of the Seismological Society of America, Vol. 99, No. 1, pp. 226-234.

Pinnegar, C. R., and D. E. Eaton (2003). Application of the S-transform to prestack noise attenuation filtering. J. Geophys. Res. , Vol.108, no. B9, 2422, doi 10.1029/2002JB00002258.

Ponzo F. C., Ditommaso R., Auletta G., Mossucca A. (2010). A Fast Method for Structural Health Monitoring of Italian Strategic Reinforced Concrete Buildings. Bulletin of Earthquake Engineering, Volume 8, Number 6, pp 1421-1434. DOI: 10.1007/s10518-010-9194-6. 
Rainieri, C.; and Fabroccino, G. (2010). Automated Output-Only Dynamic Identification of Civil Engineering Structures. Mechanical Systems and Signal Processing, 24(3): pp 678-695.

Schimmel, M., and J. Gallart (2005). The inverse S transform with windows of arbitrary and varying shape, IEEE Trans. Signal Process, 53, pp 4417-4422.

Simon, C., S. Ventosa, M. Schimmel, A. Heldring, J. J. Dañobeitia, J. Gallart, and A. Manuel (2007). The S-Transform and its inverses: side effects of discretizing and filtering. IEEE Trans. Signal Process. , Vol. 55, pp. 4928-4937, doi 10.1109/TSP.2007.897893.

Snieder Roel and Safak Erdal (2006). Extracting the Building Response Using Seismic Interferometry: Theory and Application to the Millikan Library in Pasadena, California. Bulletin of the Seismological Society of America, Apr 2006; 96: 586 - 598.

Stockwell, R. G., L. Mansinha, and R. P. Lowe (1996). Localization of the complex spectrum: the S transform. IEEE Trans. Signal Process., Vol. 44, pp. 998-1001.

Ville J. (1948). Theorie et applications de la notion de signal analytique. Cables et Transmissions, 2A, 61.

Wigner E. (1932). On the Quantum Correction For Thermodynamic Equilibrium. Phys Rev, 40, pp 749-759. 


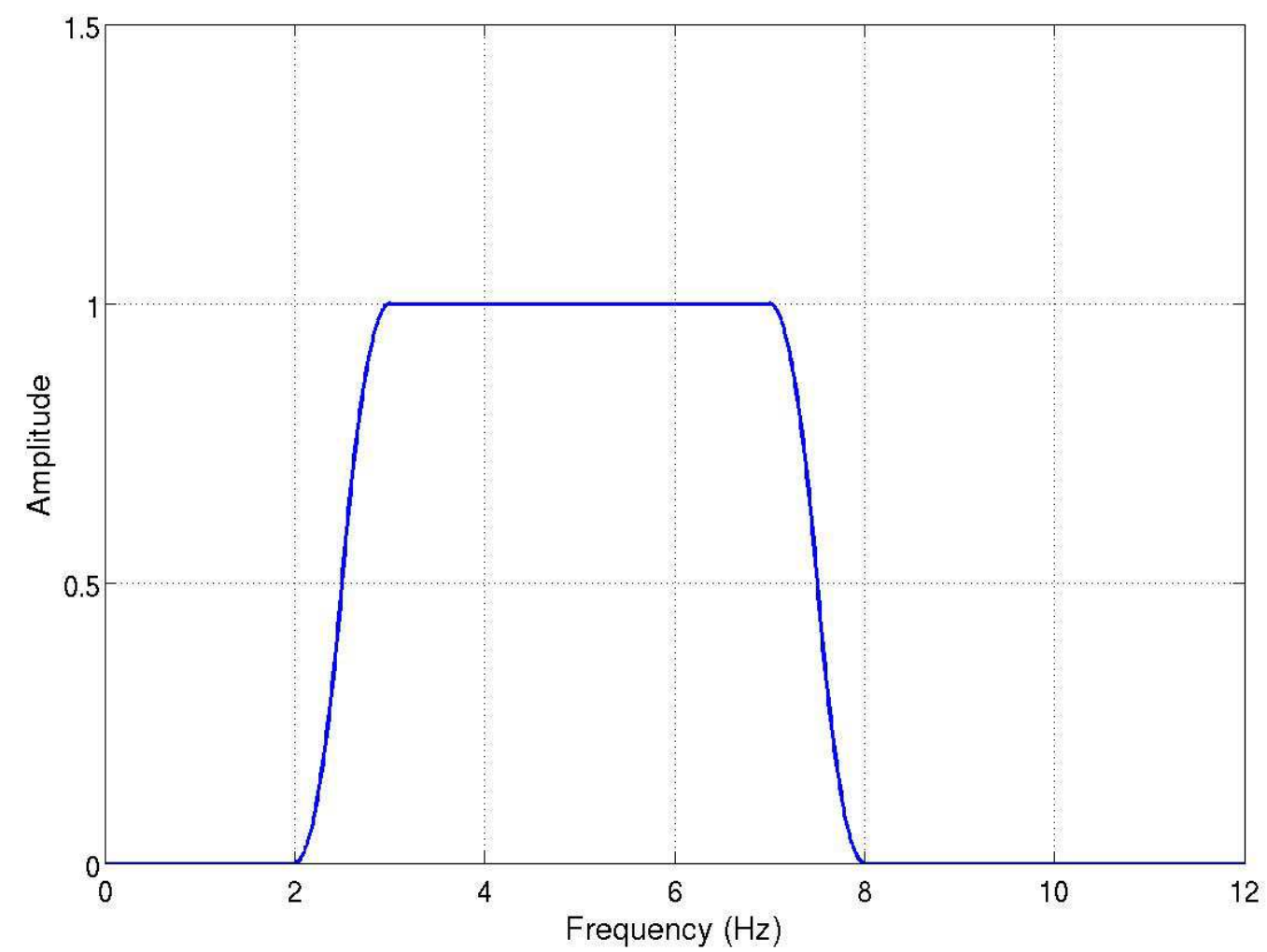

Figure 1: Example of pimf function used for build the filtering matrix $G(\tau, f)$. 


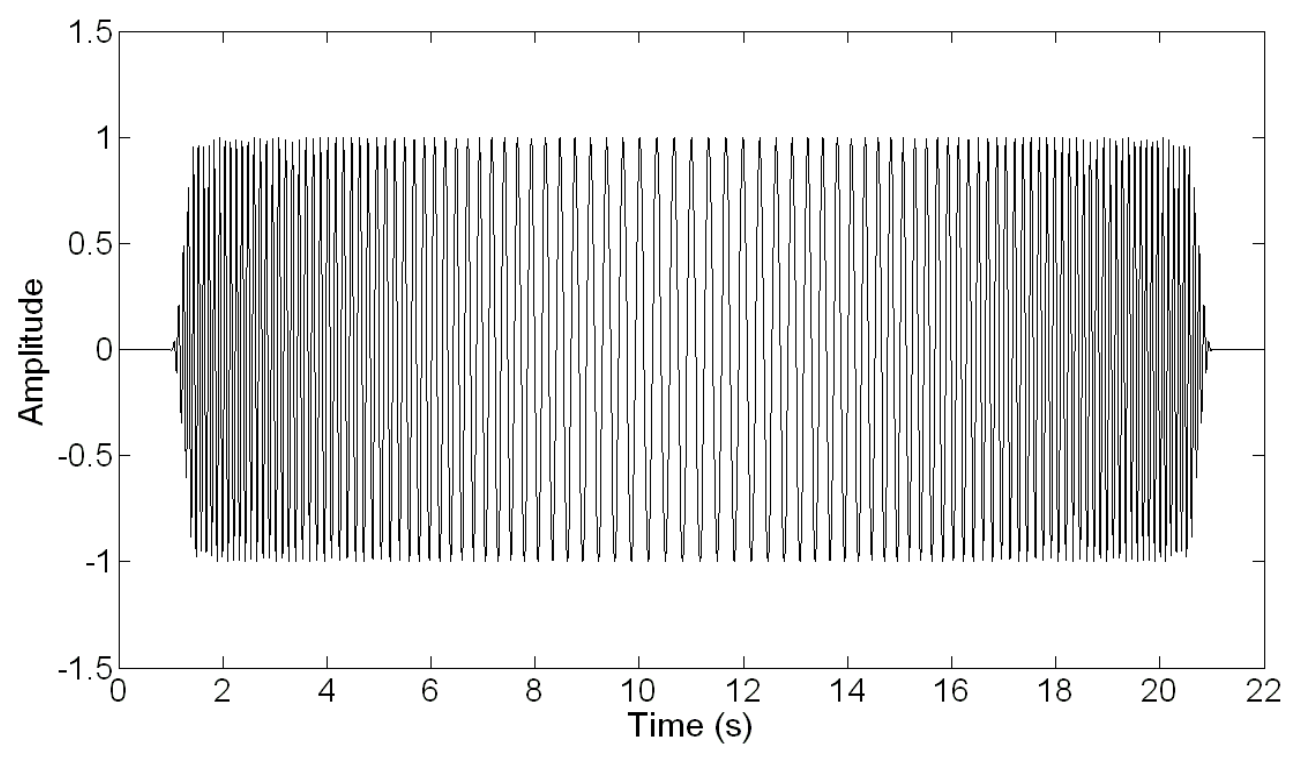

(a)

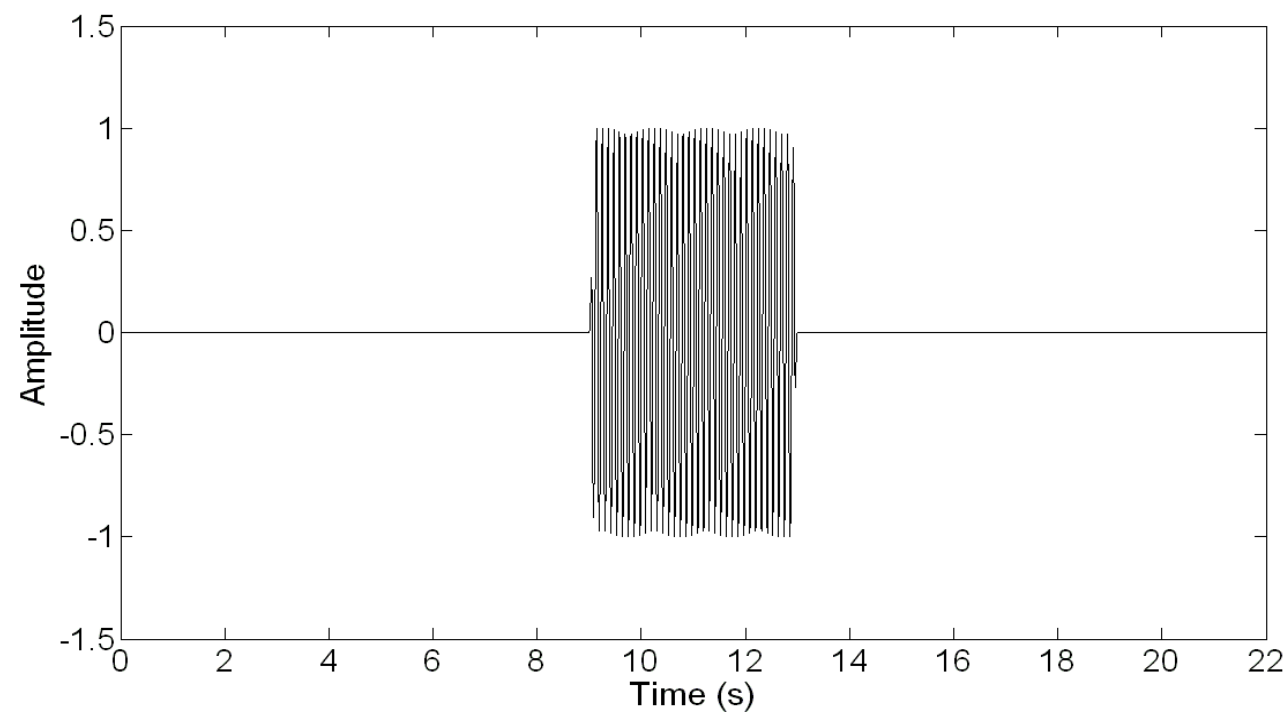

(b)

Figura 2: (a) Frequency-variable, constant amplitude sinusoid. (b) Constant-frequency, amplitude-varying sinusoid 


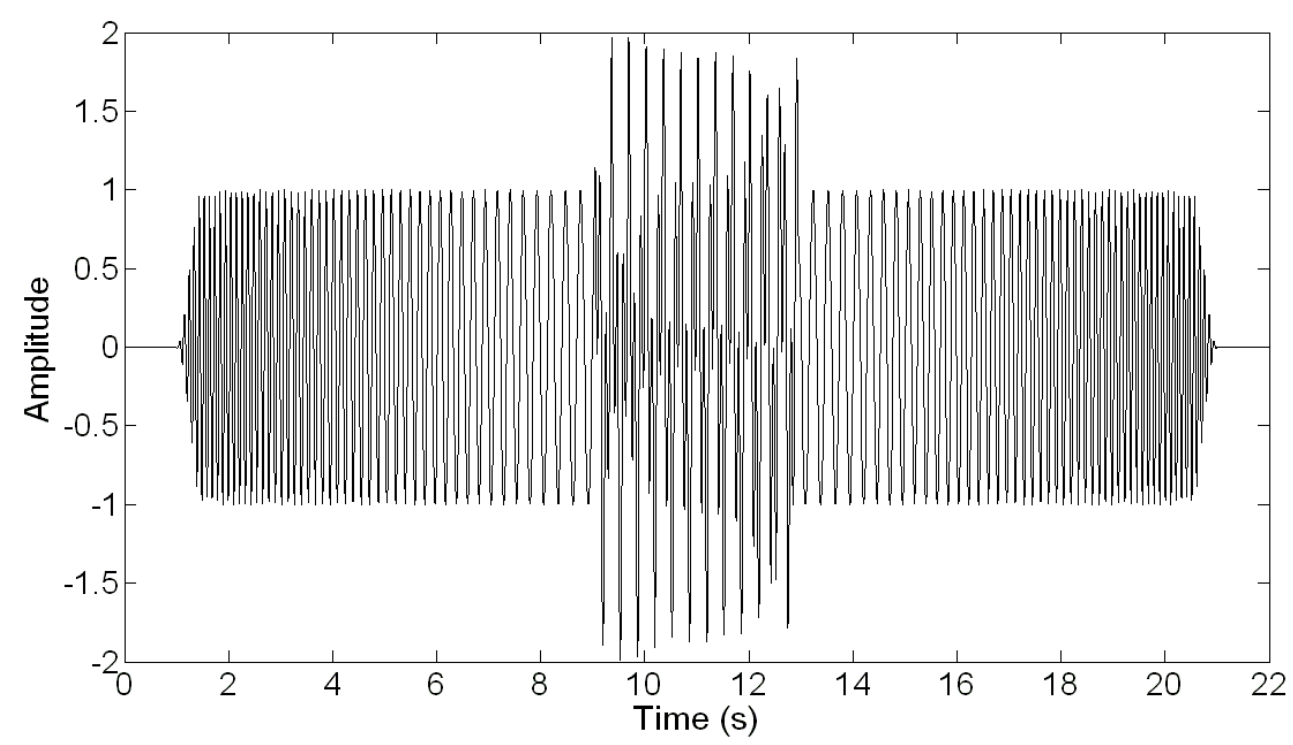

(a)

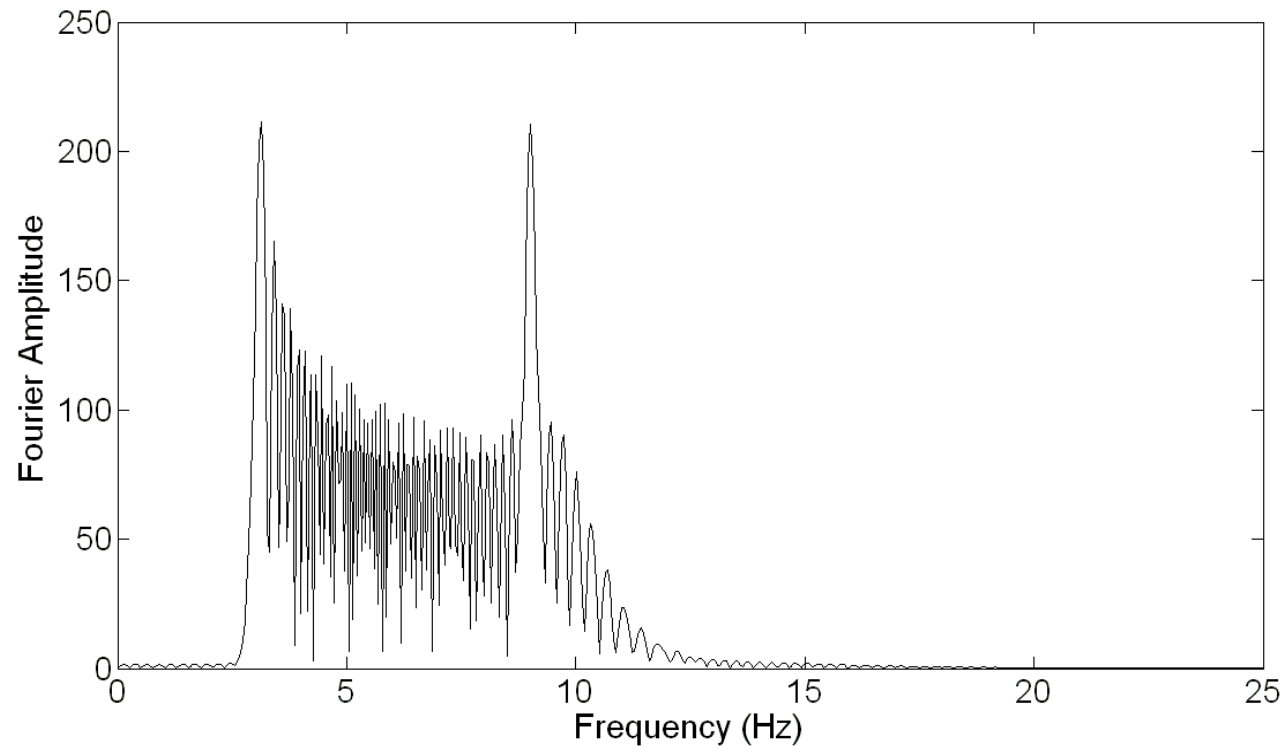

(b)

Figure 3: (a) Sum of constant-frequency sinusoid with varying ampliuude and frequency variable, amplitude-constat sinusoid. (b) Fourier spectra. 



(a)

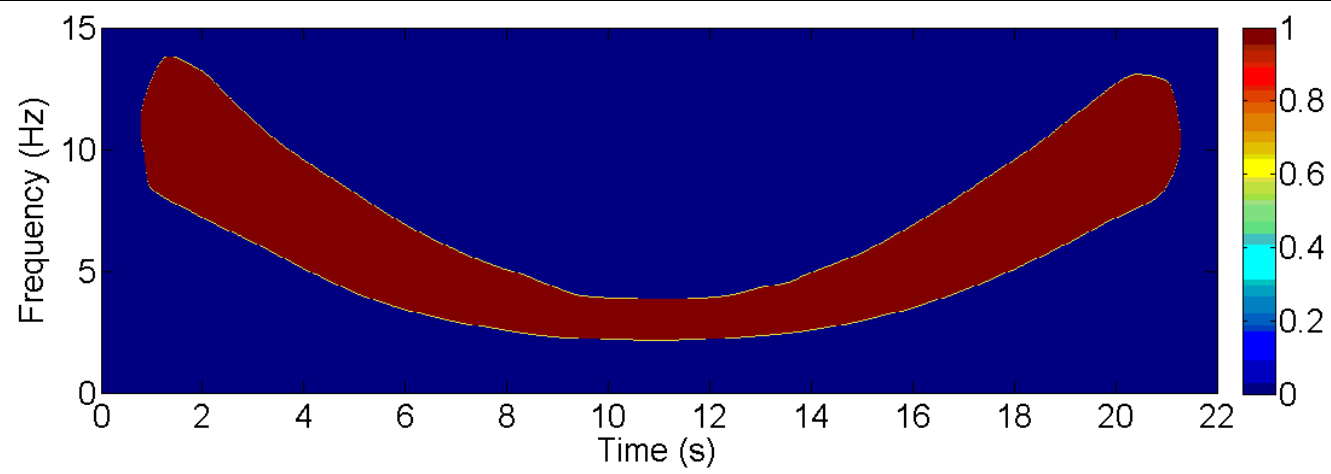

(b)
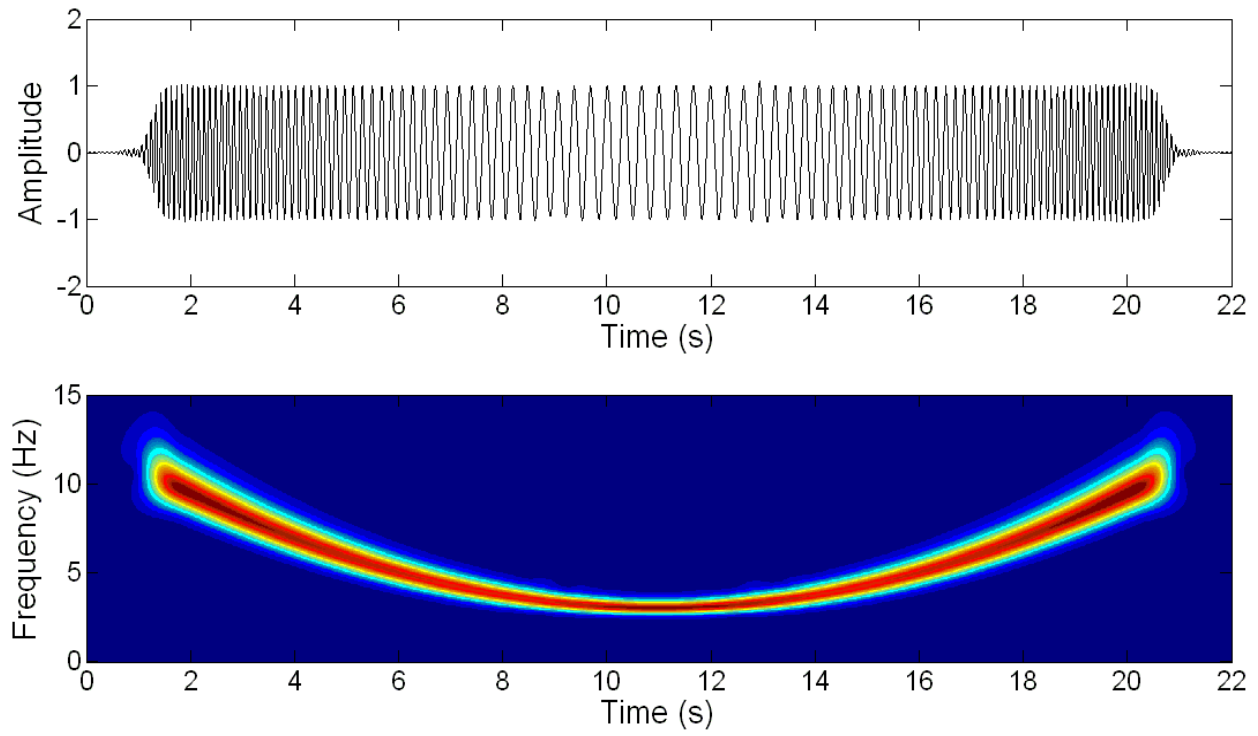

(c)

Figure 4: (a) S-Tansform of signal and selection of subdomain of interest (red curve); (b)

Filtering matrix generated by the selected subdomain; (c) Filtered signal using band-variable filter. 

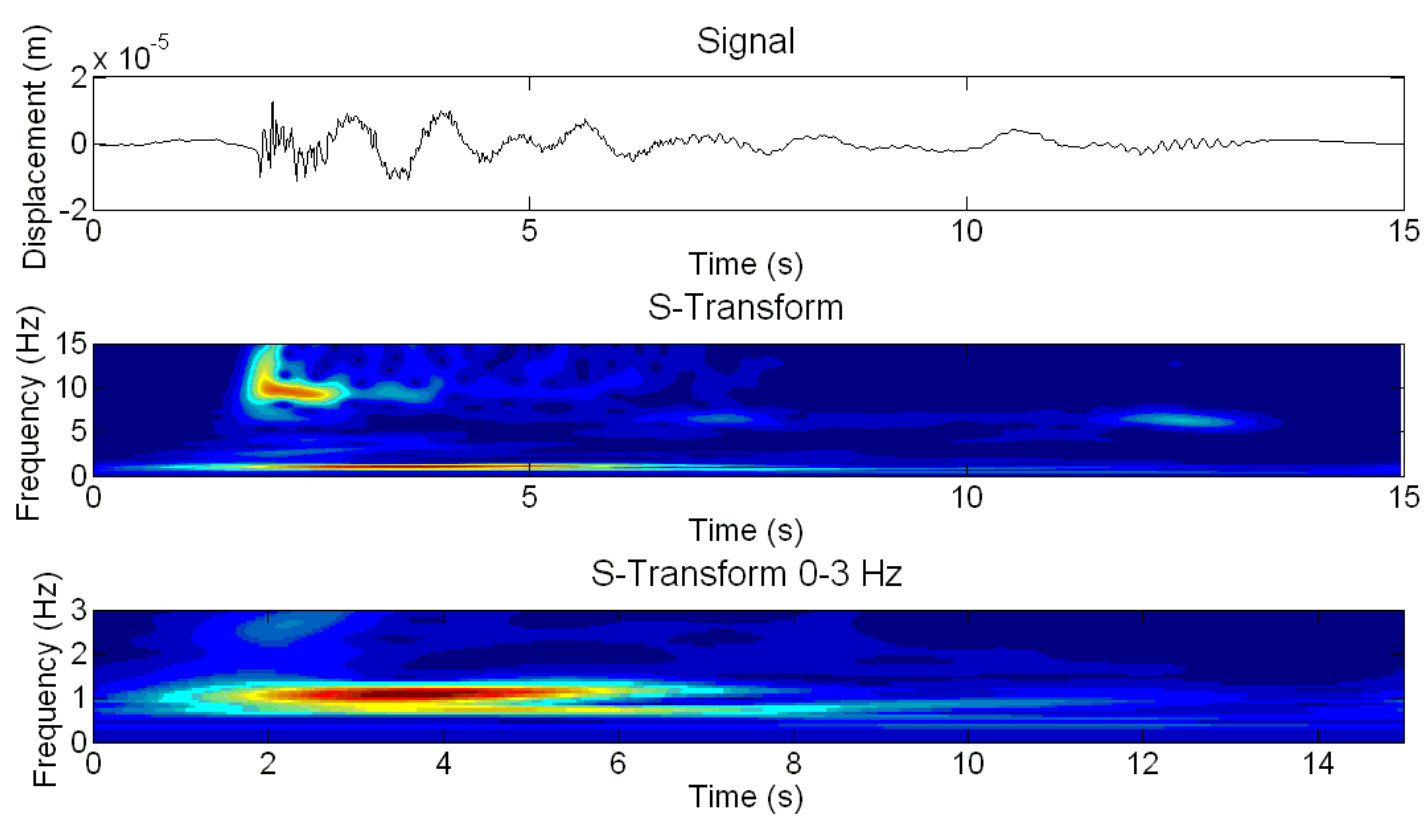

Figure 5: Free-field recording at $\mathbf{1 0} \mathrm{m}$ from building.

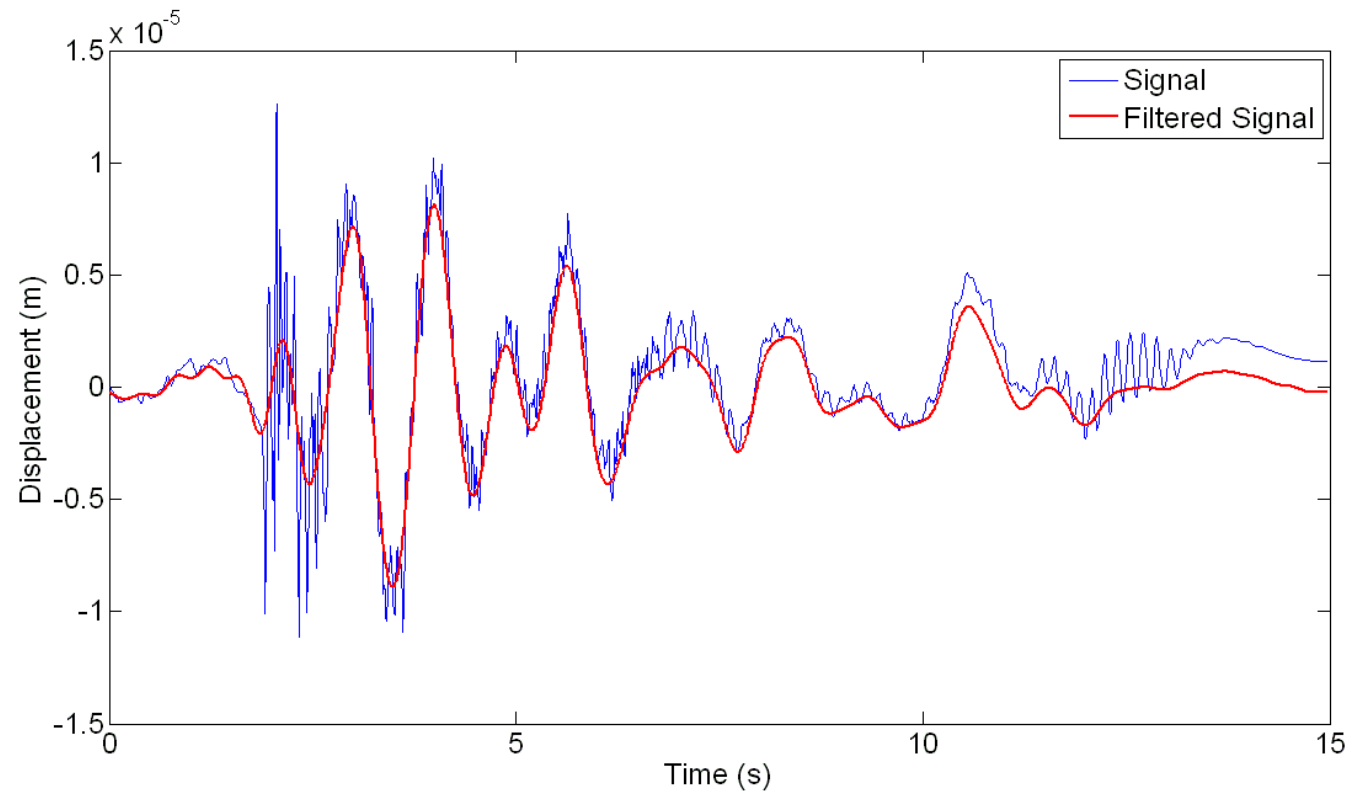

Figure 6: Comparison between filtered and original signal. 

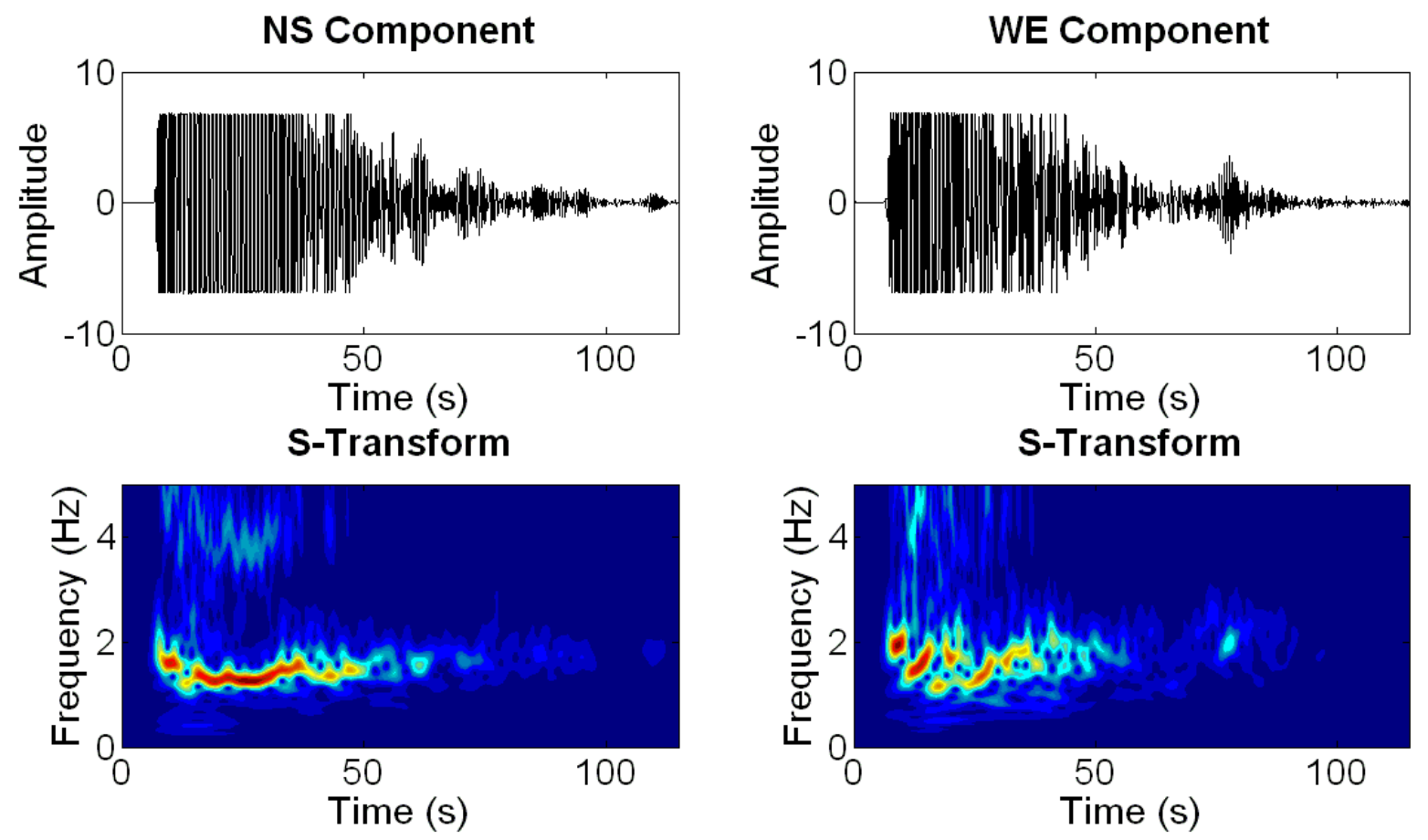

Figure 7: Velocimetric recordings within the building (Bonefro).
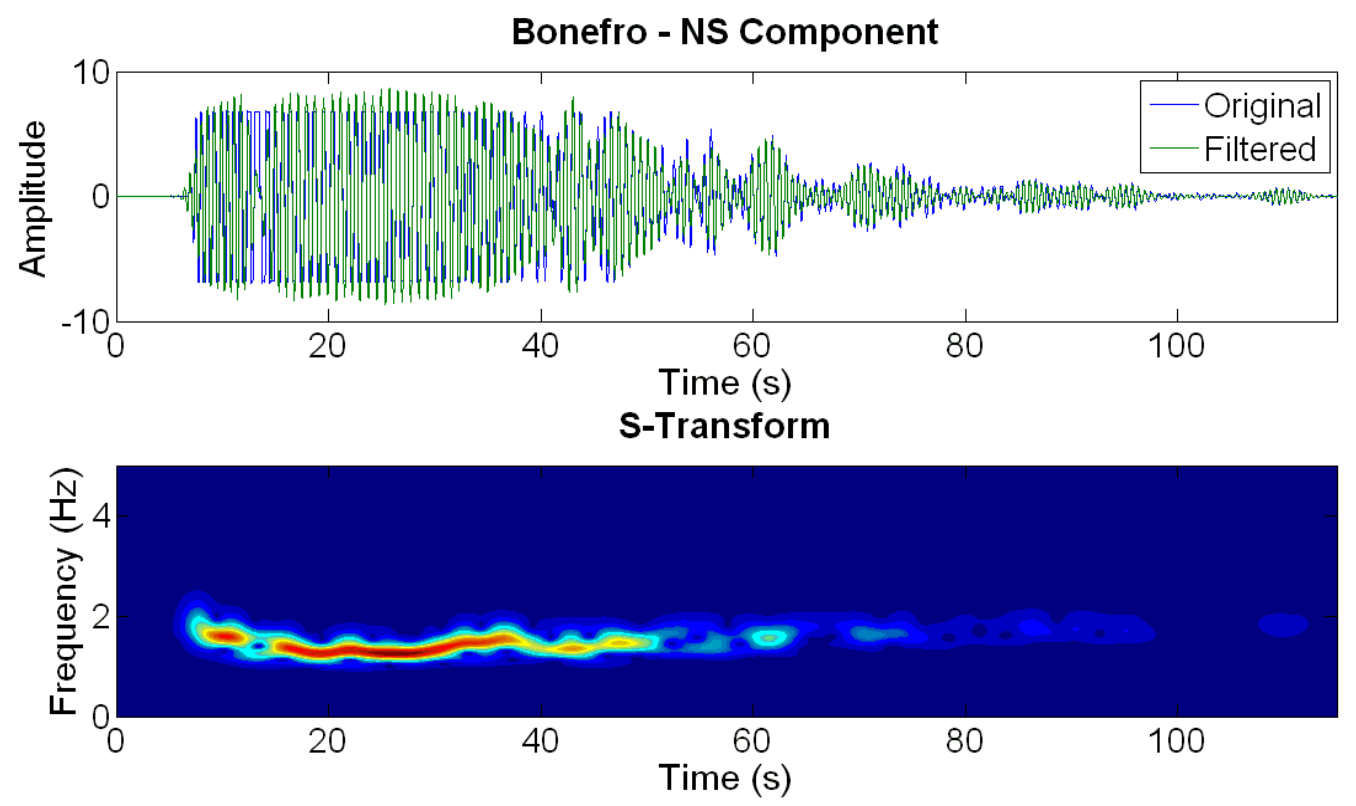

Figure 8: Extracting non-stationary behaviour of the main mode of the structure. 

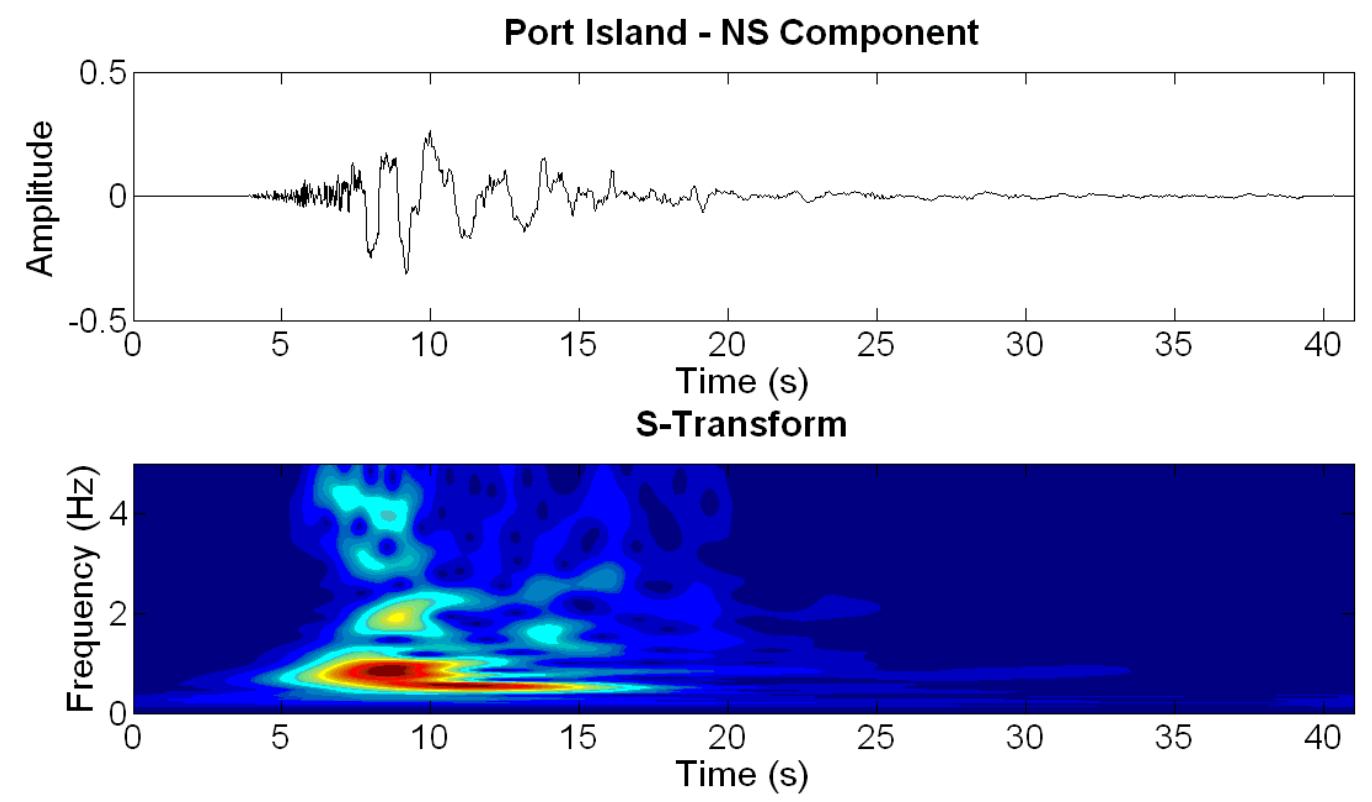

Figure 9: NS Component of Kobe earthquake recording.


Figure 10: Band-variable filter application on Kobe earthquake recording (NS component). 


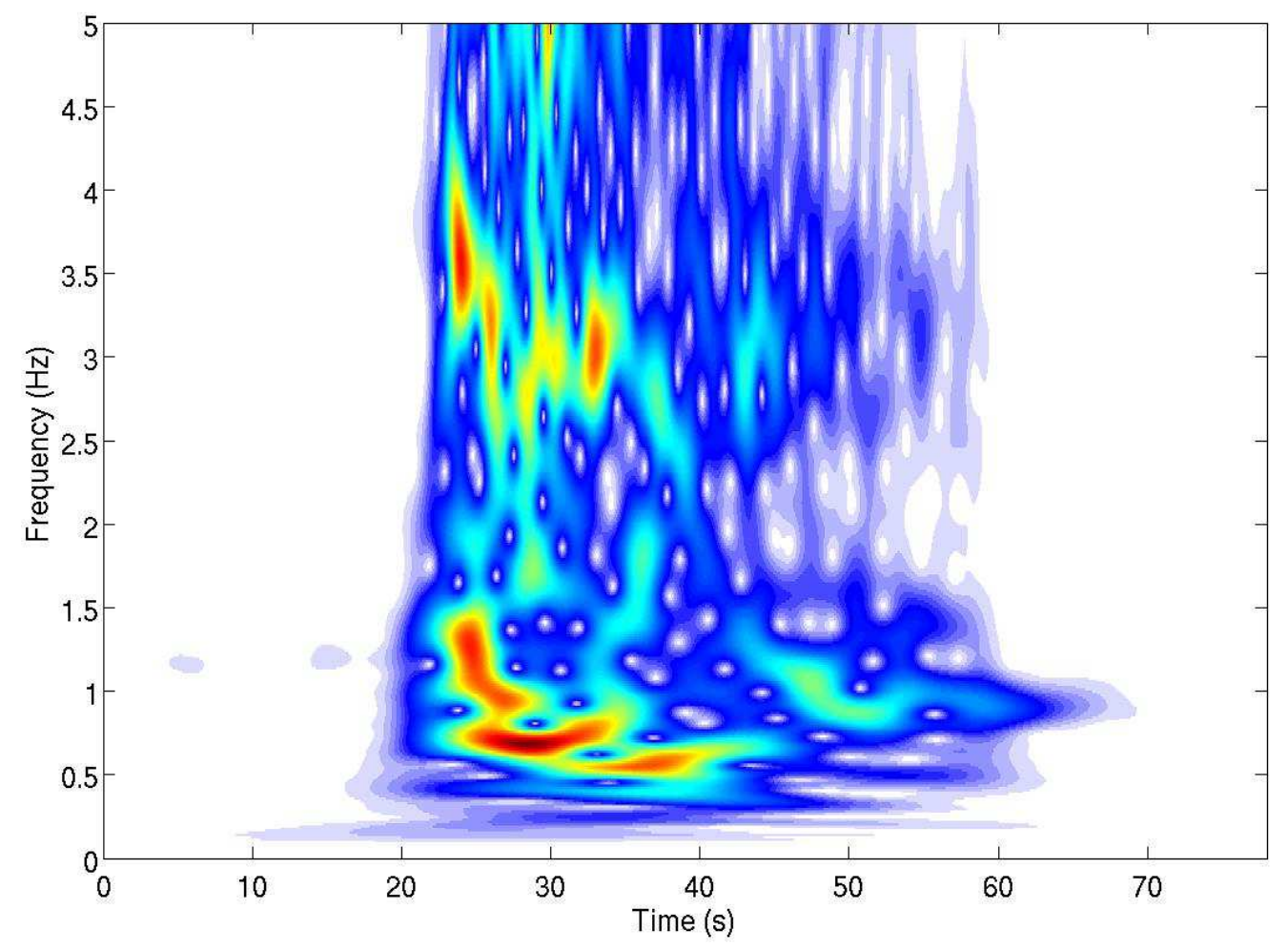

Figure 11: Numerical Model - S-Transform of the accelerometric recording of the last level

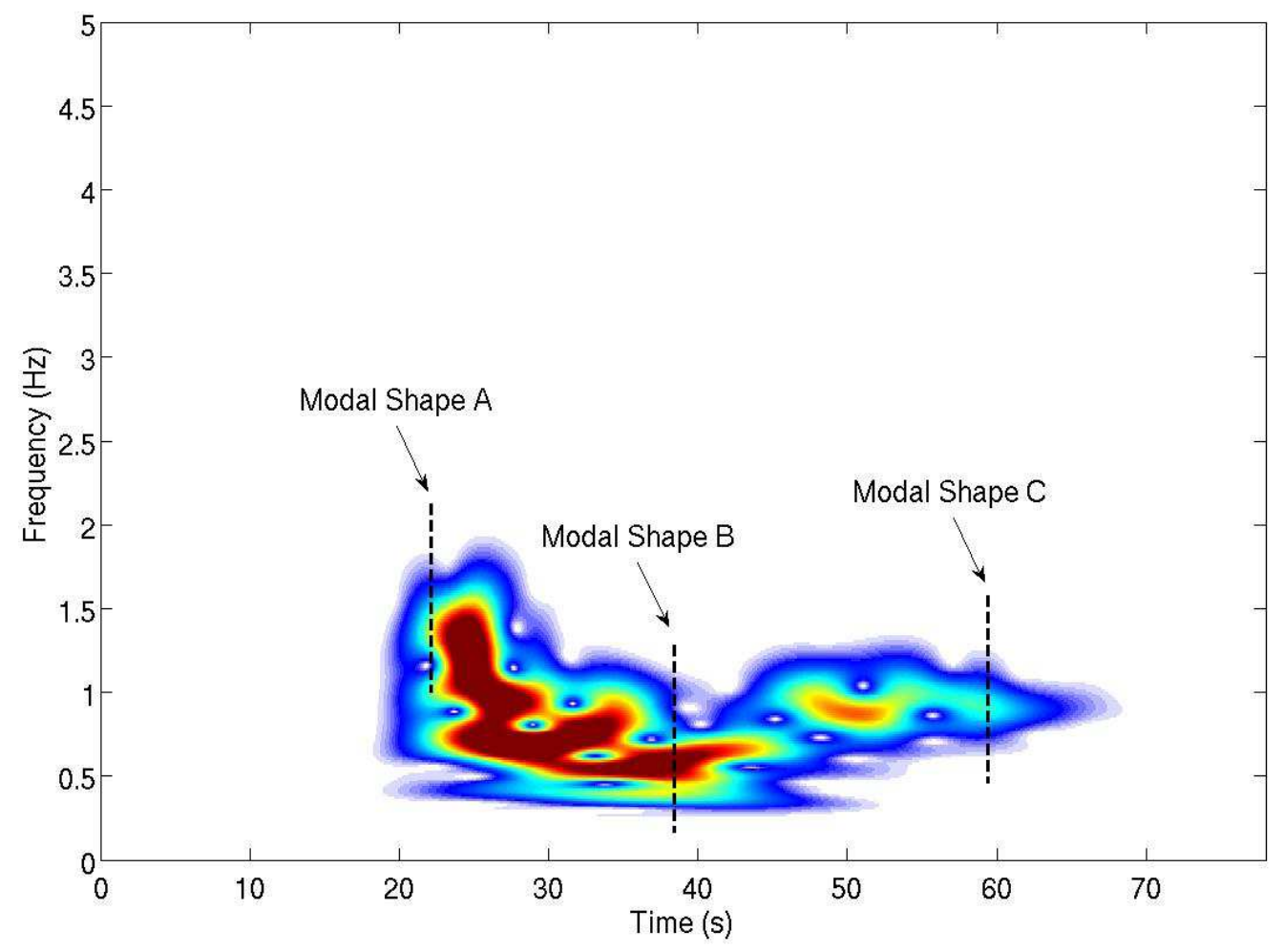

Figure 12: Numerical Model - frequency variation of the fundamental mode 



Figure 13: Numerical Model - fundamental mode shape evaluated over time 


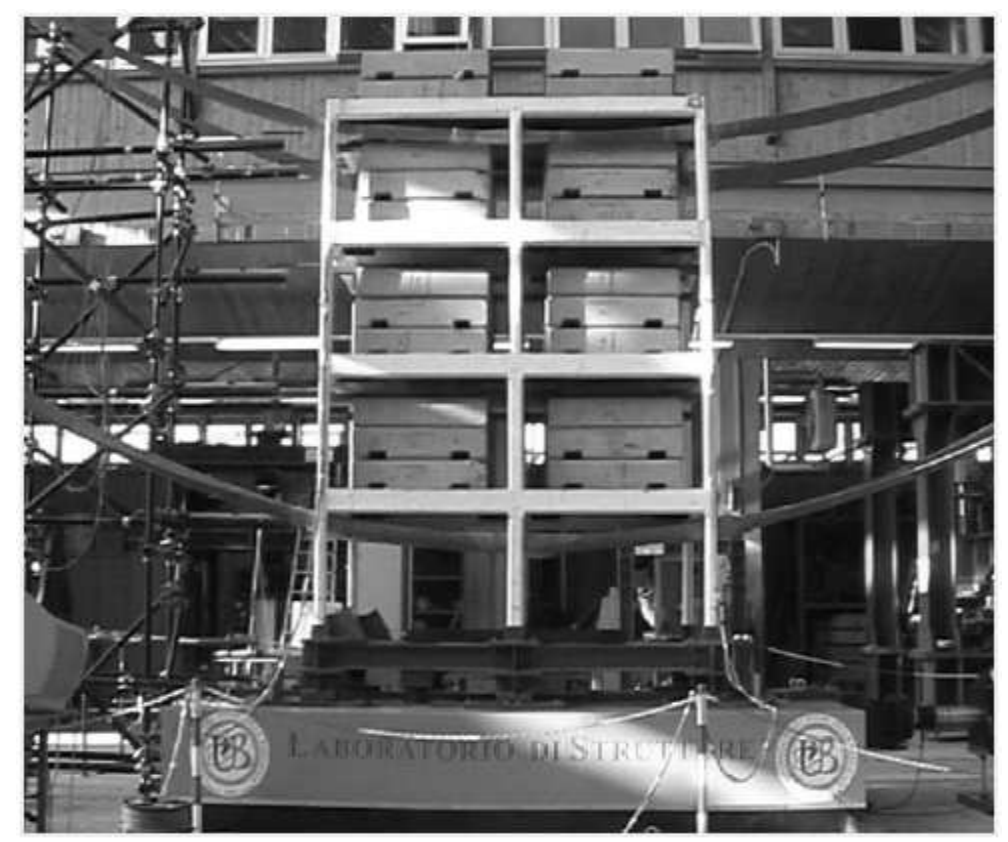

Figure 14: POP model (by Ponzo et al., 2010)

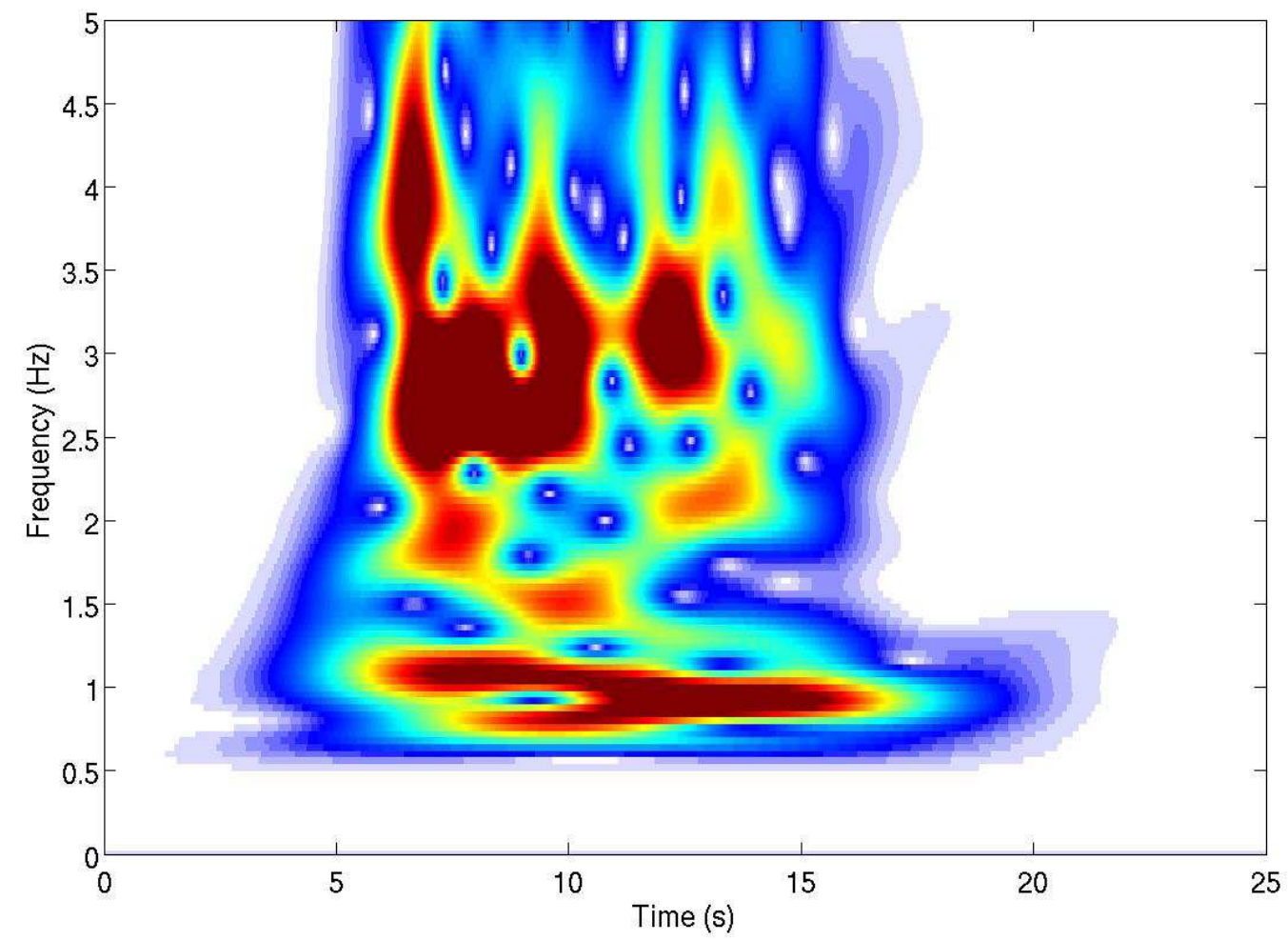

Figure 15: POP Model - S-Transform of the accelerometric recording of the last level 


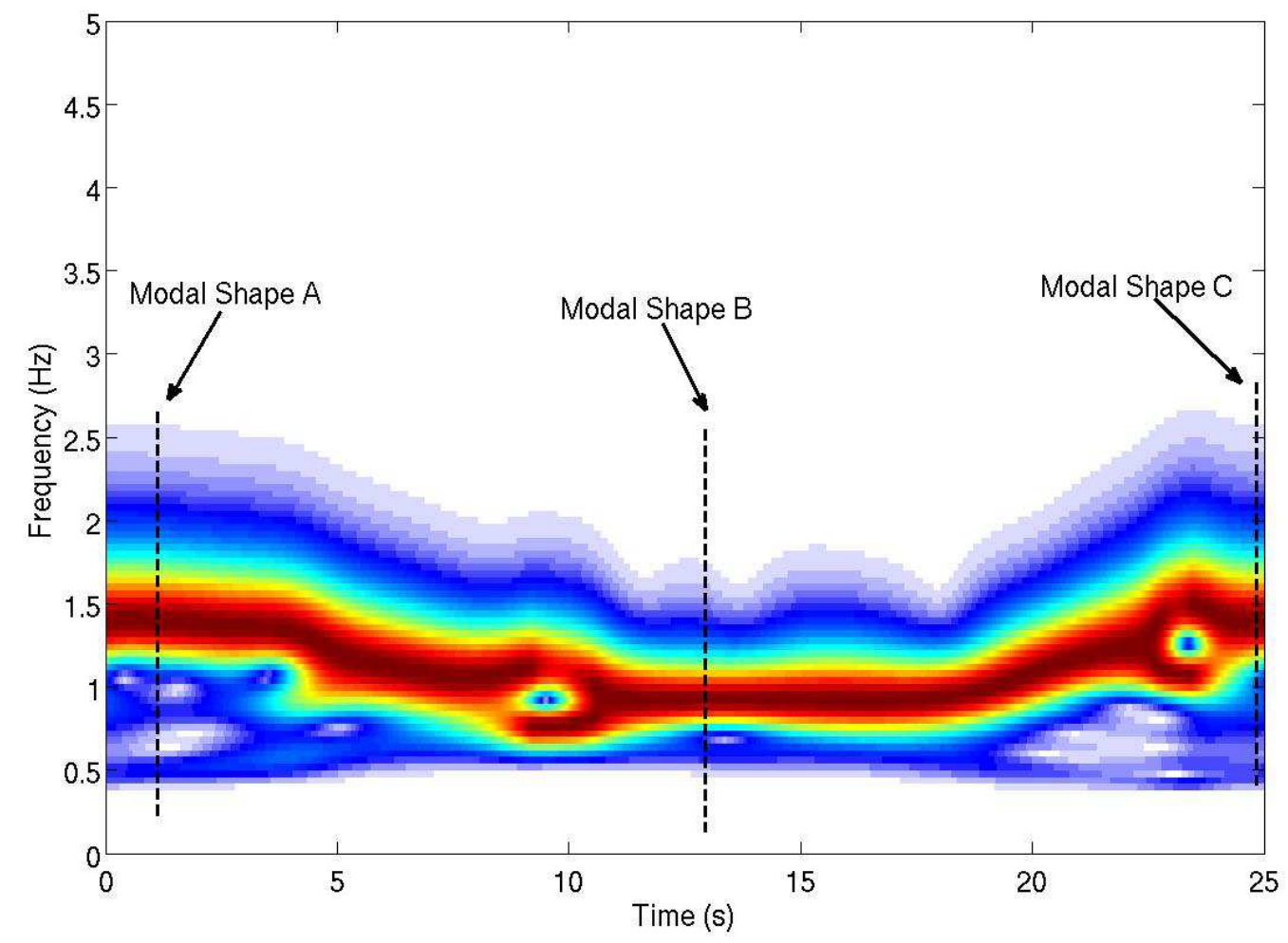

Figure 16: POP Model - frequency variation of the fundamental mode (normalized STransform)


Figure 17: POP Model - fundamental mode shape evaluated over time 